\title{
A Novel Melt Cast Composite Booster Formulation Based on DNTF/TNT/GAP-ETPE/Nano-HMX
}

\author{
Shuo Yu and Hequn Li \\ School of Chemical and Environmental Engineering, North University of China, \\ Taiyuan 030051, China \\ Correspondence should be addressed to Hequn Li; lhq6371630@163.com
}

Received 6 May 2016; Accepted 28 June 2016

Academic Editor: Ewa Schab-Balcerzak

Copyright (C) 2016 S. Yu and H. Li. This is an open access article distributed under the Creative Commons Attribution License, which permits unrestricted use, distribution, and reproduction in any medium, provided the original work is properly cited.

\begin{abstract}
To obtain the melt cast booster explosive formulation with high energy and low critical detonation diameter, melt cast explosives were designed by 3,4-bis(3-nitrofurazan-4-yl)furoxan (DNTF)/2,4,6-trinitrotoluene (TNT)/glycidyl azide polymer-energetic thermoplastic elastomer (GAP-ETPE)/nano-1,3,5,7-tetranitro-1,3,5,7-tetraazacyclooctane (HMX)/Aristowax. Furthermore, the impact sensitivity, small scale gap test, rheological properties, propagation reliability, and detonation velocity were measured and analyzed. The results show that when the mass ratio of DNTF/TNT/GAP-ETPE/nano-HMX/Aristowax is 34.2/22.8/2/40/1, not only does it indicate excellent rheological property but it has a brilliant safety performance as well. Moreover, it can propagate the detonation waves successfully in the groove at $0.7 \mathrm{~mm} \times 0.7 \mathrm{~mm}$. When the charge density in the groove is $1.70 \mathrm{~g} \cdot \mathrm{cm}^{-3}$, its detonation velocity can reach $7890 \mathrm{~m} \cdot \mathrm{s}^{-1}$.
\end{abstract}

\section{Introduction}

With the development of Micro Electromechanical System (MEMS) initiation technique, ammunition system has increasingly tended to be miniaturized, complicated, and intelligent. This requires booster explosives to possess low critical detonation diameter to meet explosive reliability in the small size channel $[1,2]$. Usually, the press mounting method has been adopted to charge for traditional booster explosives such as PBXN-5 [3], LX-14 [4], and LX-19 [5]. However, this method is not applied in the complex and small size channel. Nowadays, micro injection process was used to charge the micro channel. By injection molding technology the non-Newtonian fluid PBXlinebreak explosive slurries based on pentaerythritol tetranitrate (PETN), 1,3,5-trinitroperhydro-1,3,5-triazine (RDX), 1,3,5,7-tetranitro-1,3,5,7-tetraazacyclooctane (HMX), or hexanitro-2,4,6,8, 10,12-hexaazaisowurtzitane (CL-20) were squeezed into an irregular-shaped channel [6-9]. Although some progresses have been made in formulations, there is a lot of work to do to achieve a high energy and safety one.

The flow characteristic of melt cast explosive offers the applied possibility to the complex and micro channel.
Nevertheless, the traditional melt cast carrier TNT cannot be used alone in the micro channel for its limitations of energy, sensitivity, and critical detonation diameter. To improve the energy of melt cast explosive, 3,4-bis(3-nitrofurazan-4yl)furoxan (DNTF), which possessed high energy, low melting point, and low critical detonation diameter, was added into TNT by Xi'an Modern Chemistry Research Institute [10]. An energetic binder glycidyl azide polymer (GAP) possesses high heat of formation, high burning rate, low signature, and low shock sensitivity. As a binder it can meet the requirement of high energy and being insensitive to propellants and ammunition. Ampleman prepared energetic thermoplastic elastomer (ETPE) based on GAP. GAP-ETPE was dissolved into Composition B to obtain a greener insensitive melt cast explosive with excellent mechanical properties. In addition, GAP-ETPE can efficiently control exudation of TNT [11]. Moreover, it is well known that nano-HMX granules are more favored over the large particles in the aspect of design formulation with low critical detonation diameter [2] and mechanical sensitivity [12]. Herein, taking advantage of the outstanding characteristics of DNTF, TNT, GAPETPE, and nano-HMX, a novel melt cast composite booster 
TABLE 1: Formulations of melt cast explosive used in this experiment.

\begin{tabular}{lccccc}
\hline \multirow{2}{*}{ Samples } & \multicolumn{5}{c}{ Contents (\%) } \\
& DNTF & TNT & GAP-ETPE & Nano-HMX & Aristowax \\
\hline 1 & 28.2 & 18.8 & 2 & 50 & 1 \\
2 & 34.2 & 22.8 & 2 & 40 & 1 \\
3 & 40.2 & 26.8 & 2 & 30 & 1 \\
4 & 46.2 & 30.8 & 2 & 20 & 1 \\
\hline
\end{tabular}

formulation was prepared based on them. Furthermore, the impact sensitivity, small scale gap test, rheological properties, propagation reliability, and detonation velocity of the booster were investigated in detail.

\section{Materials and Methods}

2.1. Materials. DNTF and TNT were purchased from Gansu Yinguang Chemical Industry Co. Ltd. of China; nano-HMX granules $(80-130 \mathrm{~nm})$ were provided by North University of China [12]; GAP, $M t=3502$, with hydroxyl value of $30.41 \mathrm{mgKOH} \cdot \mathrm{g}^{-1}$, is from Luzhou North Chemical Industry Co. Ltd. of China; 1,4-butanediol (BDO) is from Tianjin Beichen Founder Reagent Factory of China, Analytical Reagent; diphenyl-methane-diisocyanate (MDI) is from Yantai Wanhua Polyurethane Co. Ltd. of China, Analytical Reagent; Aristowax is from Shanghai Chuangzhen Chemical Co. Ltd. of China; N,N-dimethylformamide (DMF) is from Tianjin Tianda Chemical Factory of China.

2.2. Synthesis of GAP-ETPE. First, GAP was put in a vacuum drying oven $(-0.08 \mathrm{MPa})$ and then dried at $100^{\circ} \mathrm{C}$. Second, GAP (10.00 g) and warmed MDI (2.02 g) were placed in a $100 \mathrm{~mL}$, three-necked, round-bottomed flask. Together they reacted at $60^{\circ} \mathrm{C}$ while stirring. After 2 hours, warmed BDO $(0.48 \mathrm{~g})$ was introduced into the mix. Third, DMF was used to dissolve the product for the next 2 hours. Finally, the solution was put into the vacuum oven $(-0.08 \mathrm{MPa})$ at $90^{\circ} \mathrm{C}$ for 7 days and then GAP-ETPE binder was obtained.

2.3. FT-IR Characterization of GAP-ETPE. PerkinElmer Spectrum 100 FT-IR spectrometer, which was made by PerkinElmer in the US, was used to characterize GAP-ETPE. The sample was carried out in a $\mathrm{KBr}$ pellet by a MCT detector at a stand-off distance of $5 \mathrm{~m}$ using a mid-IR supercontinuum light source. It was measured in $4000-400 \mathrm{~cm}^{-1}$ wave number range with a $4 \mathrm{~cm}^{-1}$ resolution.

2.4. Preparation of Melt Cast Explosive Formulations. DNTF/ TNT was selected as melt cast carrier and the mass ratio of them is 60/40 [13]. Nano-HMX granules were added as high energy solid loading explosive. GAP-ETPE and Aristowax were considered as the binder and the desensitizer, respectively. The melt cast explosives were prepared according to the formulations in Table 1.

2.5. Impact Sensitivity Test. The ERL type 12 drop hammer apparatus was used for conducting the impact sensitivity test

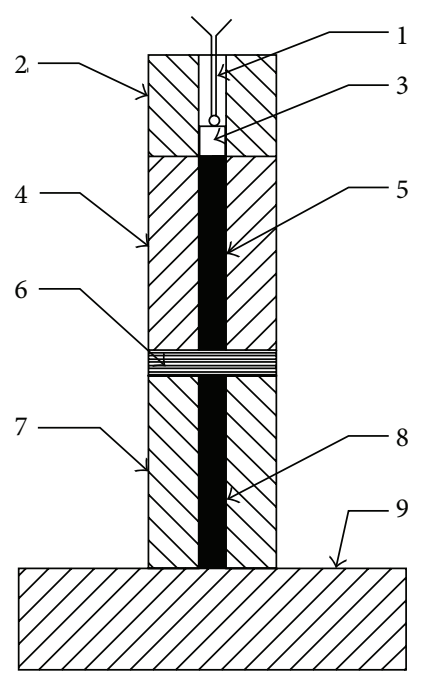
(1) Detonating fuse head
(2) Detonator seat
(3) Detonator
(4) Sleeve
(5) Donor charge
(6) Card gap (10 mm)
(7) Sleeve
(8) Boosters charge
(9) Witness plates

FIGURE 1: Schematic cross section of the experimental arrangement used to small scale gap test.

according to the GJB 772A-97 standard method 601.3 [14]. The testing conditions consisted of a drop weight of $2.500 \pm$ $0.002 \mathrm{~kg}$, a sample mass of $35 \pm 1 \mathrm{mg}$, and a relative humidity of $50 \%$. The critical drop height of $50 \%$ explosion probability $\left(\mathrm{H}_{50}\right)$ was used to represent the test results. The higher $\mathrm{H}_{50}$ is, the lower impact sensitivity is.

2.6. Small Scale Gap Test. The small scale gap test was carried out according to test method of safety for booster explosive GJB-2178.1A [15]. The test assembly was shown in Figure 1. First of all, shock wave was generated by the explosion of standard donor explosive column. Then the shock wave was attenuated by the card gap. Finally, the attenuated shock wave acted on the test sample. The test was repeated 20 times. The results were expressed by explosion probability $(P)$.

2.7. Rheological Properties Test. The R/S Plus rheometer, which was manufactured by Brookfield Ltd. of USA, was used to test the rheological properties of melt cast formulations by constant rotation measurement unit. The testing conditions were temperature, $80^{\circ} \mathrm{C}$; shearing rate, $2 \mathrm{~s}^{-1}$; measure points, 60 ; testing time, $60 \mathrm{~s}$.

2.8. Propagation Reliability Test. The booster explosives were injected into the grooves of $0.7 \mathrm{~mm} \times 0.7 \mathrm{~mm}$. The detonation reliability test was conducted for the charge according to Figure 2. 


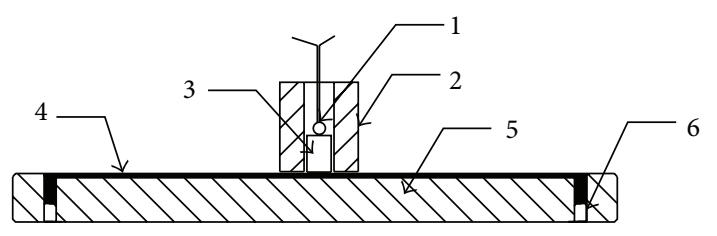
(1) Detonating fuse head
(2) Detonator seat
(5) Aluminum plates
(3) Detonator
(4) Booster explosive charge in groove

FIGURE 2: Diagrammatic sketch of propagation reliability test.

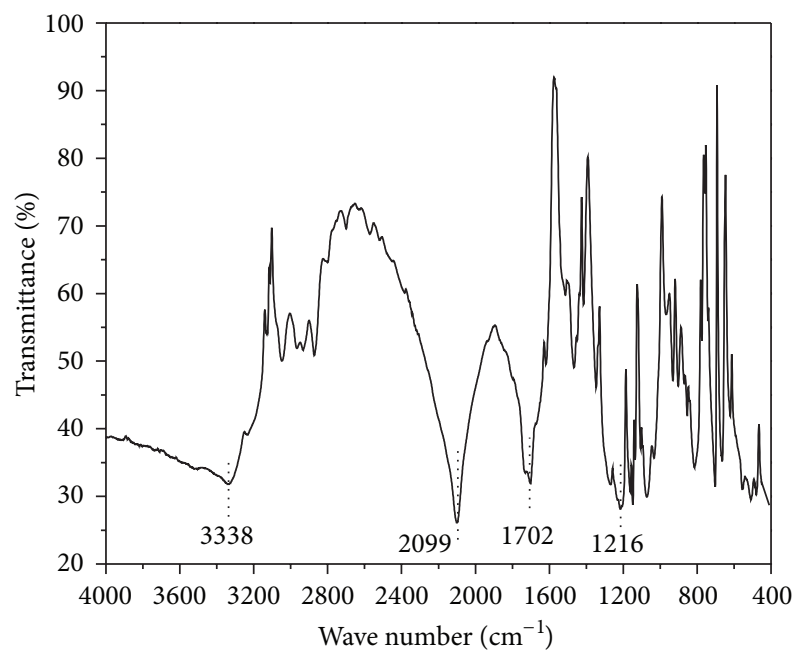

FIgURE 3: FT-IR spectrum of GAP-ETPT.

2.9. Detonation Velocity Test. The detonation velocity in groove booster explosive was measured using the probing method. The sensor probe was installed onto the surface of the charging groove, and the distance of test points was controlled with vernier caliper. The detonation propagation time was measured by oscilloscope.

\section{Results and Discussion}

3.1. FT-IR of GAP-ETPE. The FT-IR spectrum of GAP-ETPE was illustrated in Figure 3. In the FT-IR image, the characteristic bands at approximately 3338,1702 , and $1216 \mathrm{~cm}^{-1}$ confirm the presence of urethane grouping-NHCOO-because of the stretching vibration of $\mathrm{N}-\mathrm{H}$, stretching vibration of $\mathrm{C}=\mathrm{O}$, and asymmetric stretching vibration of C-O-C. In addition, the band at about $2099 \mathrm{~cm}^{-1}$, which represents an absorption peak of $-\mathrm{N}_{3}$, indicates that GAP-ETPE possesses energetic characteristics.

3.2. Formulations Detonation Velocity Estimation. The detonation velocities of samples were estimated by EXPLO5 v6.01 computer program [16]. The results were shown in Table 2. The densities of these four melt cast formulations are more than $1.8 \mathrm{~g} \cdot \mathrm{cm}^{-3}$. Their detonation velocities are higher than
TABLE 2: Results of detonation velocity estimation.

\begin{tabular}{lcc}
\hline Samples & Density $\left(\mathrm{g} \cdot \mathrm{cm}^{-3}\right)$ & Detonation velocity $\left(\mathrm{m} \cdot \mathrm{s}^{-1}\right)$ \\
\hline DNTF & 1.937 & 9502 \\
TNT & 1.654 & 7241 \\
HMX & 1.905 & 9234 \\
ETPE & 1.293 & 6645 \\
Aristowax & 1.090 & 6451 \\
1 & 1.830 & 8647 \\
2 & 1.821 & 8562 \\
3 & 1.813 & 8485 \\
4 & 1.804 & 8409 \\
\hline
\end{tabular}

TABLE 3: Impact sensitivity and small scale gap test results of samples.

\begin{tabular}{lcc}
\hline Samples & $\begin{array}{c}\text { Impact sensitivity, } \\
\mathrm{H}_{50}(\mathrm{~cm})\end{array}$ & $\begin{array}{c}\text { Small scale gap } \\
\text { test, } P(\%)\end{array}$ \\
\hline Raw DNTF & 25.3 & 100 \\
Raw TNT & 149.6 & 0 \\
Nano-HMX & $47.3[12]$ & 15 \\
1 & 58.6 & 0 \\
2 & 50.2 & 0 \\
3 & 48.3 & 0 \\
4 & 47.3 & 10 \\
\hline
\end{tabular}

$8000 \mathrm{~m} \cdot \mathrm{s}^{-1}$. From the perspective of energy output, these four melt cast formulations may serve as high energy boosters.

3.3. Impact Sensitivity and Small Scale Gap Test. Under mechanical impact and shock waves action, most of the mechanical energy of the explosive transfers to thermal energy firstly. Due to the asymmetry of the mechanical impact and shock waves, instead of acting on the whole explosive, the thermal energy only concentrates on the local scope and forms hot spots. The explosive at the hot spot has thermal decomposition in the first place and releases heat at the same time, which prompts the decomposition speed acceleration of the explosive. If the hot spot number formed in the explosive is enough and the size is large enough, after the hot spot temperature rises to the bursting point, the explosive will be stimulated at these spots and have explosion. Finally, it causes the explosion of partial or even the whole explosive. The impact sensitivity and small scale gap test results of samples are demonstrated in Table 3. From Table 3 the impact sensitivity of formulation decreases as nano-HMX content increasing and DNTF content decreasing. This is because the impact sensitivity of nano-HMX is lower than that of DNTF. Among them, formulation 4 has not passed the small scale gap test of booster. As a result, formulations 1, 2, and 3 can be chosen as boosters in terms of sensitivity.

3.4. Rheological Properties Analysis. The influence of nanoHMX content on viscosity of booster formulations was shown in Figure 4. It is apparent from Figure 4 that, with nano-HMX content increasing, the viscosity of formulation increases. 


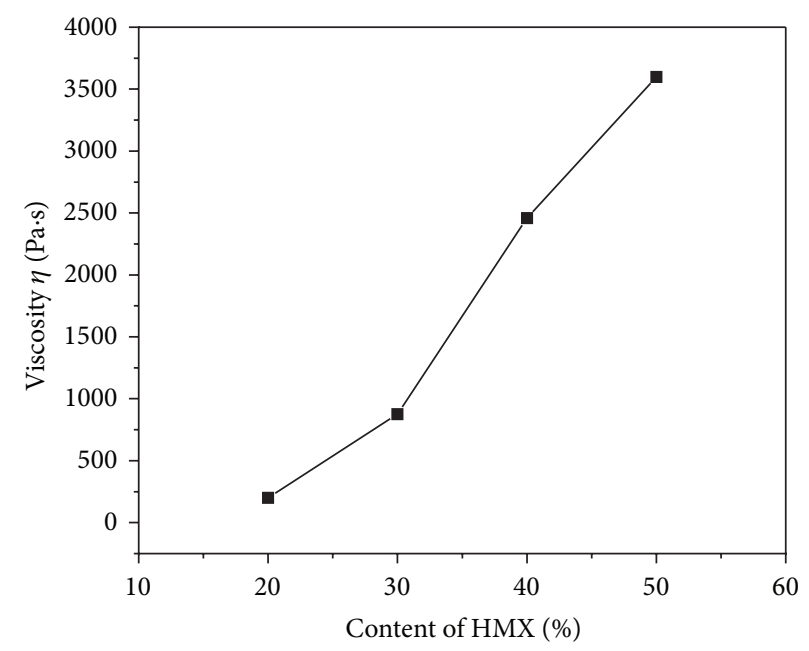

FIGURE 4: Influence of nano-HMX content on viscosity of booster formulations.

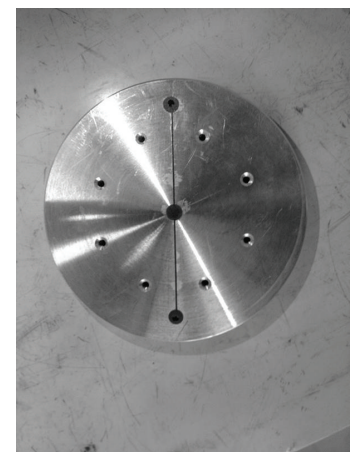

(a)

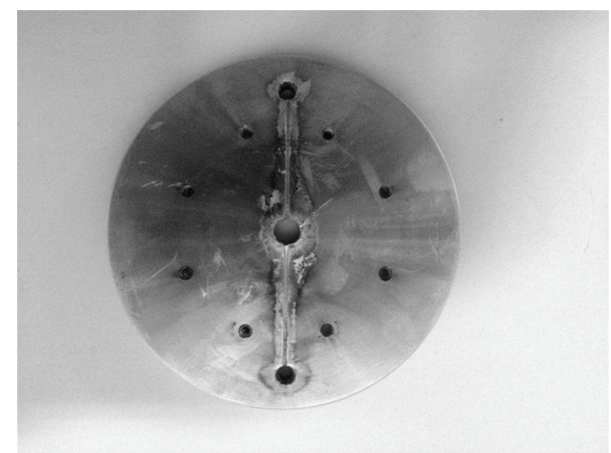

(b)

FIGURE 5: Groove charge and postdetonation aluminum witness plates: (a) groove charge and (b) detonation propagation.

When nano-HMX content reaches 50\%, the viscosity of formulation adds up to above $3500 \mathrm{~Pa} \cdot \mathrm{s}$. Therefore, formulation 1 is not applicable to a micro injection process for its poor flow ability. Formulations 2, 3, and 4 can be selected as candidates for booster explosives.

3.5. Propagation Reliability and Detonation Velocity. The propagation reliability test is an important factor to determine a new booster explosive. Formulation 2 turns out well in the sight of energy output, sensitivity, and viscosity. The result of propagation reliability test of formulation 2 is shown in Figure 5. When groove size of aluminum witness plate is $0.7 \mathrm{~mm} \times 0.7 \mathrm{~mm}$, formulation 2 can propagate the detonation waves successfully. The density of charge in the groove $(0.7 \mathrm{~mm} \times 0.7 \mathrm{~mm})$ is $1.70 \mathrm{~g} \cdot \mathrm{cm}^{-3}$ (93.4\% TMD). The theoretical and measured detonation velocity values are 8562 and $7890 \mathrm{~m} \cdot \mathrm{s}^{-1}$. This leads us to conclude that the critical detonation diameter of formulation 2 is less than $0.7 \mathrm{~mm}$ and it has high energy characteristics.

\section{Conclusions}

A booster explosive based on DNTF/TNT/GAP-ETPE/nanoHMX/Aristowax with the mass ratio $34.2 / 22.8 / 2 / 40 / 1$ has been prepared successfully by a melt cast process. First, the booster explosive exhibits good sensitivity properties, for its drop height is $50.2 \mathrm{~cm}$ and it has passed the small scale gap test. Second, this booster shows good flow ability in that its viscosity is less than $2500 \mathrm{~Pa} \cdot \mathrm{s}$. Finally, the critical detonation diameter of this melt cast booster formulation is less than $0.7 \mathrm{~mm}$ and when the density of the charge is $1.70 \mathrm{~g} \cdot \mathrm{cm}^{-3}$ (93.4\% theoretical maximum density), its measured detonation velocity can reach $7890 \mathrm{~m} \cdot \mathrm{s}^{-1}$. By virtue of these outstanding properties, the melt cast booster explosive is expected to be a candidate filled in the complex and micro channel. By adjusting the formulations the booster explosive with higher energy, less sensitivity, and lower critical detonation diameter may be obtained.

\section{Competing Interests}

The authors declare that they have no competing interests.

\section{References}

[1] C. Rossi, K. L. Zhang, D. Estève, P. Alphonse, P. Tailhades, and C. Vahlas, "Nanoenergetic materials for MEMS: a review," Journal of Microelectromechanical Systems, vol. 16, no. 4, pp. 919-931, 2007. 
[2] I. F. Kobylkin, "Critical detonation diameter of highly desensitized low-sensitivity explosive formulations," Combustion, Explosion and Shock Waves, vol. 45, no. 6, pp. 732-737, 2009.

[3] P. W. Chen, F. L. Huang, K. D. Dai, and Y. S. Ding, "Detection and characterization of long-pulse low-velocity impact damage in plastic bonded explosives," International Journal of Impact Engineering, vol. 31, no. 5, pp. 497-508, 2005.

[4] D. M. Hoffman, "Fatigue of LX-14 and LX-19 plastic bonded explosives," Journal of Energetic Materials, vol. 18, no. 1, pp. 127, 2000.

[5] R. L. Simpson, P. A. Urtiew, D. L. Ornellas, G. L. Moody, K. J. Scribner, and D. M. Hoffman, "CL-20 performance exceeds that of HMX and its sensitivity is moderate," Propellants, Explosives, Pyrotechnics, vol. 22, no. 5, pp. 249-255, 1997.

[6] B. W. Harris, "Oil/High explosive compatibility study. Selection of safing fluids for damaged explosives assemblies," Propellants, Explosives, Pyrotechnics, vol. 9, no. 1, pp. 7-11, 1984.

[7] D. S. Wulfman, O. Sitton, F. T. Nixon et al., "Reformulation of solid propellants and high explosives: an environmentally benign means of demilitarizing explosive ordnance," The Canadian Journal of Chemical Engineering, vol. 75, no. 5, pp. 899-912, 1997.

[8] K. Wu, Y.-C. Liu, D.-C. Liu, J.-M. Yuan, and J.-H. Wang, "Study on the extrusion-cast booster and charging process for the small grooved channel," Acta Armamentarii, vol. 34, no. 2, pp. 251256, 2013.

[9] J. Y. Wang, C. W. An, G. Li, L. Liang, W. Z. Xu, and K. Wen, "Preparation and performances of castable HTPB/CL-20 booster explosives," Propellants, Explosives, Pyrotechnics, vol. 36, no. 1, pp. 34-41, 2011.

[10] Q. H. Wang, "A new melt-cast explosive formulation," Chinese Journal of Energetic Materials, vol. 12, no. 1, pp. 46-55, 2004.

[11] G. Ampleman, P. Brousseau, S. Thiboutot et al., "Evaluation of GIM as a greener insensitive melt-cast explosive," International Journal of Energetic Materials and Chemical Propulsion, vol. 11, no. 1, pp. 59-87, 2012.

[12] C. W. An, H. Q. Li, W. J. Guo, X. H. Geng, and J. Y. Wang, "Nano cyclotetramethylene tetranitramine particles prepared by a green recrystallization process," Propellants, Explosives, Pyrotechnics, vol. 39, no. 5, pp. 701-706, 2014.

[13] H.-Q. Li, C.-W. An, W.-J. Guo, Y.-J. Wei, H.-X. Li, and J.-Y. Wang, "Study on rheological properties of DNTF/TNT/HMX based on melt-cast explosive," Journal of North University of China (Natural Science Edition), vol. 35, no. 6, pp. 720-728, 2014.

[14] National Military Standard of China, Explosive Test Method GJB 772A-97, 1997 (Chinese).

[15] National Military Standard of China, Test Method of Safety for Booster Explosive GJB-2178.1A, 2005 (Chinese).

[16] M. Sućeska, "Calculation of the detonation properties of C-HN-O explosives," Propellants, Explosives, Pyrotechnics, vol. 16, no. 4, pp. 197-202, 1991. 

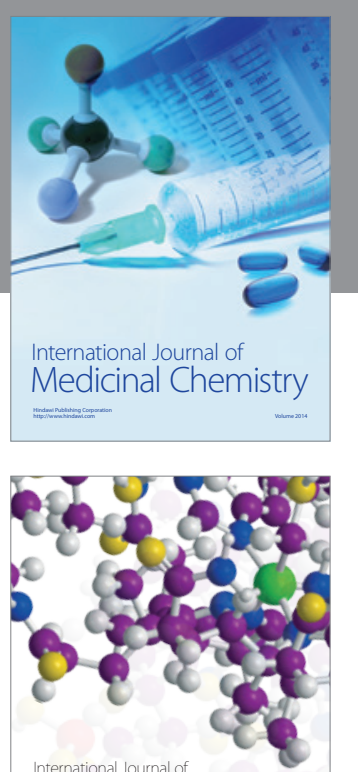

Carbohydrate Chemistry

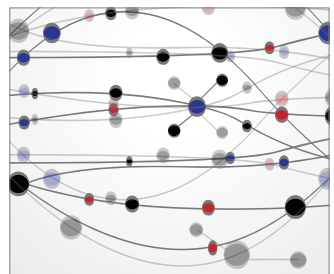

The Scientific World Journal
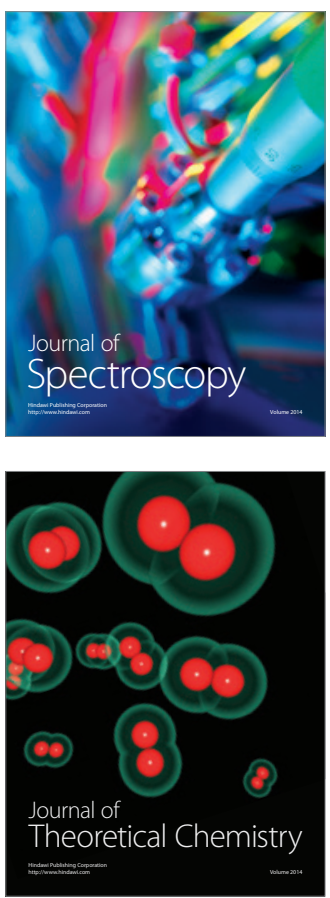
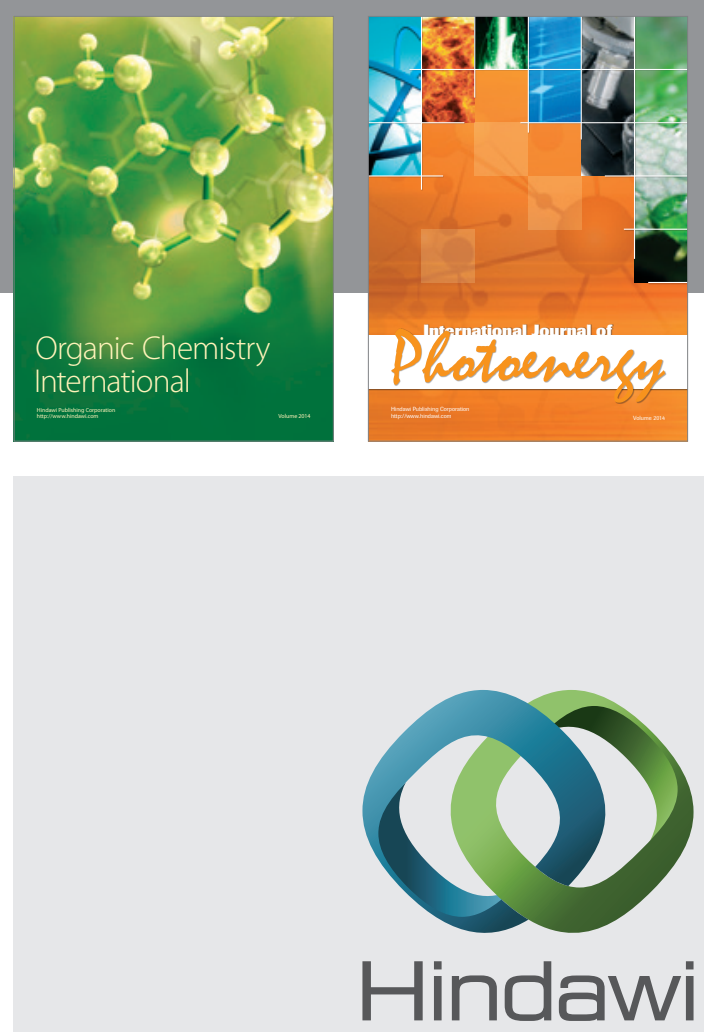

Submit your manuscripts at

http://www.hindawi.com

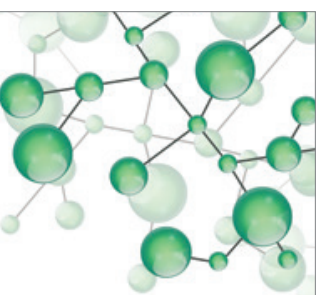

International Journal of

Inorganic Chemistry

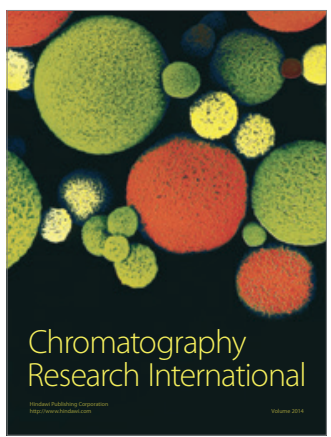

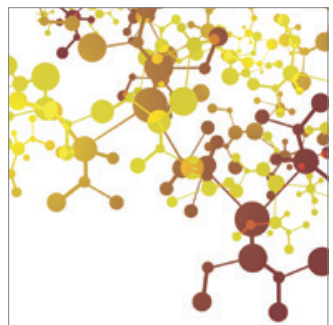

Applied Chemistry
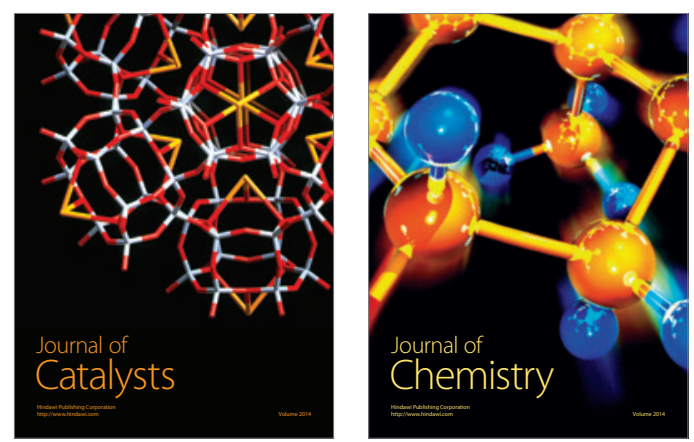
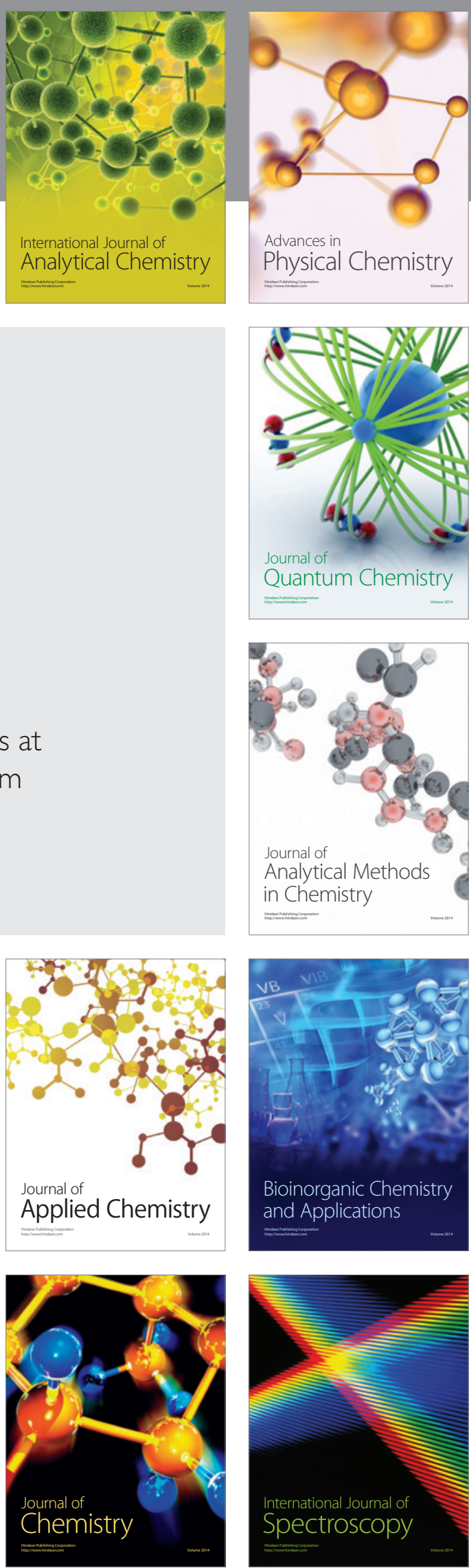